\title{
PET/CT Imaging of Lymphoma Outside the Western World
}

\author{
Raoul Gabus ${ }^{1}$ - Astrid Pavlovsky ${ }^{2} \bullet$ Martin Eleta $^{3} \bullet$ Tetiana Skrypets $^{4} \bullet$ \\ Irina Kryachok ${ }^{5} \bullet$ Olga Novosad $^{5} \bullet$ Yana Stepanishyna $^{5} \bullet$ \\ Yaroslav Kmetyuk ${ }^{6}$ - Ahmed Alaskar ${ }^{7,9,10} \bullet$ Naila Shaheen $^{9,10}$ • Ali SA 8,9,10 • \\ Mubarak Al-Mansour $7,9,10$ • Fabrizio Bergesio ${ }^{11}$ • Stephane Chauvie ${ }^{11}$ \\ ${ }^{1}$ Hospital Maciel, Montevideo, Uruguay; ${ }^{2}$ Fundaleu, GATLA, Buenos Aires, Argentina; \\ ${ }^{3}$ Imaxe Imaging center, Buenos Aires, Argentina; ${ }^{4} \mathrm{PhD}$ Program in Clinical and \\ Experimental Medicine, University of Modena and Reggio Emilia, Reggio Emilia, Italy; \\ ${ }^{5}$ Oncohematology Department, National Cancer Institute, Kyiv, Ukraine; ${ }^{6}$ Nuclear \\ Medicine Department, Clinical Hospital "Feofaniya", Kyiv, Ukraine; ${ }^{7}$ Department of \\ Oncology, Ministry of the National Guard - Health Affairs, Saudi Arabia; ${ }^{8}$ Department of \\ Radiology, Ministry of the National Guard - Health Affairs, Saudi Arabia; ${ }^{9}$ King Abdullah \\ International Medical Research Center, Saudi Arabia; ${ }^{10}$ College of Medicine (COM), King \\ Saud Bin Abdulaziz University for Health Sciences (KSAU-HS), Saudi Arabia; \\ ${ }^{11}$ Medical Physics Department S. Croce Hospital, Cuneo, Italy
}

Author for correspondence: Stephane Chauvie, Medical Physics department, S. Croce Hospital, Cuneo, Italy. Email: chauvie.s@ospedale.cuneo.it

Cite this chapter as: Gabus R, Pavlovsky A, Eleta M, Skrypets T, Kryachok I, Novosad O, Stepanishyna Y, Kmetyuk Y, Alaskar A, Shaheen N, Ali SA, Al-Mansour M, Bergesio F, Stephane Chauvie S. PET Imaging of Lymphoma Outside the Western World. In: Gallamini A, Juwied M. editors. Lymphoma. Brisbane (AU): Exon Publications. Online first 2021 Nov 21.

Doi: https://doi.org/10.36255/exon-publications.lymphoma.2021.pet-imaging

Abstract: In 2014, a committee of clinicians and nuclear medicine experts issued the recommendations for lymphoma staging and restaging based on the use of positron emission tomography/computed tomography (PET/CT). These recommendations were immediately adopted in the Western countries and have

In: Gallamini A, Juwied M (Editors). Lymphoma. Exon Publications, Brisbane, Australia. ISBN: 978-0-6453320-0-1; Doi: https://doi.org/10.36255/exon-publications.lymphoma.2021

Copyright: The Authors.

License: This open access article is licenced under Creative Commons Attribution-NonCommercial 4.0 International (CC BY-NC 4.0) https://creativecommons.org/licenses/by-nc/4.0/ 
become part of the routine clinical use. In contrast, the adoption of these recommendations has been gradual in many non-Western countries, and around the world in general, depending on the availability of dedicated and skilled technicians and doctors, and on the accessibility to economic resources in the healthcare system for the reimbursement of the cost of the procedure. This chapter presents a portrait of the adoption and accessibility of PET/CT in Uruguay, Argentina, Ukraine, and Saudi Arabia. The chapter concludes with a section on the reproducibility of SUV-related indexes for PET/CT reporting in lymphoma.

Keywords: PET/CT for lymphoma in Uruguay; PET/CT for lymphoma in Argentina; PET/CT for lymphoma in Ukraine; PET/CT for lymphoma in Saudi Arabia; SUV-related indexes for PET/CT

\section{INTRODUCTION}

The Lugano classification for initial evaluation, staging, and response assessment of Hodgkin and non-Hodgkin lymphoma has become a standard practice in the management of lymphoma (1). It is based on the use of Positron Emission Tomography/Computed Tomography (PET/CT). Since its introduction in 2014, most developed Western countries quickly adopted the recommendations, but this has not been the case for many non-Western countries. The adoption has been gradual, depending on the availability of technical expertise and economic resources. In this chapter, the authors report their experience on the use of PET/ $\mathrm{CT}$ for the overall management of lymphoma in their respective countries. In the first section, the authors describe the healthcare regulation to access the different applications of PET scanning according to the lymphoma subtype and disease status in Uruguay. In the second section, Argentinian onco-hematologists and nuclear medicine physicians jointly report their experience of PET scanning in Hodgkin Lymphoma (HL) and also the original data generated by an innovative interim PET adapted clinical trial in all-stage HL. In the third section, Ukrainian onco-hematologists and nuclear medicine physicians report the modality of PET scanning in Diffuse Large B-Cell Lymphoma (DLBCL) as an archetype of availability and accessibility to PET scanning in all the lymphoma subsets in their country. They focus on the peculiarity of the healthcare system in Ukraine and their struggle for a public coverage of the cost related to a standard use of PET scan in ${ }^{18} \mathrm{~F}$-fluorodeoxyglucose (FDG)-avid lymphoma in their country, according to the international guidelines. In the fourth section, the authors portray the use of PET scanning in lymphoma in Saudi Arabia with a spotlight on the national guidelines for one of the most frequent lymphoma subsets, the follicular lymphoma (FL). In the fifth section, the efforts toward the use of PET scanners in reproducible ways are described by the imaging core lab of the Italian Foundation on Lymphoma, who report their experience in the accreditation of more than 240 PET sites for clinical trials, both in western and non-western countries. 


\section{PET/CT IN LYMPHOMA STAGING AND RESTAGING IN URUGUAY}

The framework for PET/CT scanning of lymphoma in Uruguay was set in 2014. The national health authority has set a Uruguayan center for molecular imaging (CUDIM) (2) devoted to assistance, research, and training in molecular imaging that centralizes this imaging technique and concentrates the experience of image reading and reporting to satisfactorily respond to the demands of a population of three and a half million inhabitants. CUDIM is equipped with three PET/CT cameras and two cyclotrons to produce positron-emitting radiopharmaceuticals. About 4,000 ${ }^{18} \mathrm{~F}$-FDG PET/CT studies are performed annually for various oncological, neurological and cardiological indications.

Lymphomas are the most frequent clinical oncological indication, representing $25-28 \%$ of all studies with ${ }^{18} \mathrm{~F}-\mathrm{FDG}$. At this writing in October 2021, 325-350 studies for HL and 690-700 studies for non-Hodgkin Lymphoma (NHL) were performed between January 2021 to October 2021, which is a decrease of about $10 \%$ because of COVID-19 pandemic. When performed at baseline, PET/CT (bPET) allows a better display of tumor spread at diagnosis compared to standard radiological tools, especially of the disease spread to extra-nodal sites. When performed interim during treatment (i-PET) it allows a timely assessment of the response to therapy, thus allowing a treatment intensity guidance based on early response assessment. When performed at the end of treatment (f-PET) it proved a useful tool to assess with a higher overall accuracy the depth of the response. Finally, PET/CT turned out to be useful to assess the extension of disease upon disease relapse. This paradigm shift applies also, and even more, to low-resource or developing countries, to rationalize the healthcare expenses and to improve the appropriateness of therapies. In particular, PET/CT allows to maintain a traditional and low-cost therapeutic program for good-prognosis patients while guiding treatments to more aggressive and expensive drugs in the few patients with a very poor prognosis. In other words, investing in a complex but very useful diagnostic and prognostic tool in daily clinical practice, turned out as the best way to correctly allocate the available economic resources in a restricted indication for poor-prognosis patients deserving this kind of advanced but also very- expensive treatment. Despite the overall accuracy of PET/CT in treatment response assessment, this advanced imaging technique cannot replace biopsy procedures which are indicated in cases of equivocal results in end-of-treatment PET/CT, especially in case of residual FDG uptake in the context of a CT-detected residual mass.

The CUDIM participated in local research projects to reduce treatment-related morbidity in pediatric HL identifying four textural features in the bPET as candidates for predicting early response to chemotherapy at iPET (3). Analyses were used for optimal cut-off values to separate responders at iPET according to the Dauville score. The Dauville score at iPET was useful for differentiating PFS rates. The center has also participated in a multicenter prospective study for demonstrating that the use of a regular field of view (R-FOV) (from eyes to thighs) when compared to the recommended total-body FOV (from vertex to toes) is not 
inferior in defining the clinical stage of the disease or treatment definition (4). As so, R-FOV for both staging and interim PET/CT scans could be performed providing significant advantages in decreasing radiation exposure and scanning time, potentially improving image quality, and reducing anesthesia time. This consideration may be quite impactful in developing countries where there are limited pediatric PET/CT scanners available. Major strengths of this study were that this was the biggest series of cases of pediatric lymphoma patients enrolled in an international multicenter trial spanning differing socioeconomic layers as defined by the World Bank to include low-middle, upper-middle, and highincome countries.

\section{Accessibility}

Uruguay has a National Integrated Health Service (SNIS) since 2005 (5). The Comprehensive Health Care Plan (PIAS) is a catalog of benefits and programs the providers from the SNIS must provide (6). Many of the health benefits not included in the PIAS are covered by the National Resource Fund (FNR) (7). The FNR is a non-state public body regulated by law. It grants financial coverage to highly specialized medical procedures, as well as high-cost drugs, with proven effectiveness. It is governed by its own regulatory framework, thus guaranteeing accessibility to all citizens, and offers free and universal coverage, irrespective of public or private healthcare provider. Since 2010 Uruguay has incorporated the access to PET/CT scanning in a centralized way under the financial coverage of FNR. This coverage has been extended to the entire population that requires it and establishes a regulation and normative to access PET/CT scanning in the overall lymphoma management.

The adoption of a given imaging technique requires indeed well-defined regulations and strategies for indications and evaluation of responses, in order to result in a well-balanced diagnostic tool between quality, equity and sustainability. The regulatory framework is based on bibliographic reviews of available evidence of national and international recommendations, which are periodically reviewed to adapt them to the evolution of scientific evidence. The Uruguayan Group of Lymphomas (GULI) created by the Uruguayan Society of Hematology (SHU) (8) discusses and updates the diagnostic tools and therapeutic options, for both clinical practice and clinical research purposes. The FNR-funded procedures are summarized in the following sections.

\section{- Staging}

o bPET for staging purpose is indicated for patients with a confirmed diagnosis of HL or NHL. The following histological variants are excluded: Chronic Lymphocytic Leukemia/Small Lymphocytic Lymphoma, Lymphoplasmacytic Lymphoma, Waldenström's Macroglobulinemia, Mycosis Fungoides.

- Evaluation of therapeutic response

o Interim PET in HL: Patients with a confirmed diagnosis of HL after at least two cycles of chemotherapy when performed for treatment intensity adaptation.

o Interim PET in NHL: Patients with a confirmed diagnosis of NHL after at least two cycles of chemotherapy if disease progression is suspected after IHD and the results would determine a change in therapeutic behavior. 
o EoT- PET in HL and NHL: Within three months after completing the treatment appropriate to the pathology and stage of the patient. The Deauville 5-point scale is recommended as interpretation key. EoT-PET can be repeated later in case a complete remission (CR) is not reached. In patients reaching $\mathrm{CR}$ in interim or EoT-PET, follow-up with PET is not indicated.

- Re-staging

o When tumor recurrence or persistence is suspected if it determines a change in therapeutic behavior. Furthermore, the bPET study is necessary to interpret the degree of response both at EoT- and i-PET.

\section{Specific considerations according to the type of B Cell Lymphoma}

According to the Lugano2014 criteria (1,10-11), routine Bone Marrow biopsy (BMP) can be omitted if the pattern of FDG uptake is consistent with a focal infiltration. In case of a diffuse or low intensity infiltration, or negative PET, BMP is required to exclude discordant pathology or reactive infiltration. However, at this writing in October 2021, the normative in Uruguay for Diffuse large B-Cell Lymphoma (DLBCL) is mandatory trephine BMP to assess the presence of disease in this anatomic district. Although the time of i-PET after the 2 nd or 4 th cycle is considered, there are studies that show greater diagnostic profitability after the $4^{\text {th }}$ treatment cycle (12-14). But treatment escalation cannot be recommended in routine clinical practice based on iPET, except in those cases in which disease progression is confirmed $(13,14)$. iPET assessment should be performed using the visual Deauville scale. EOT PET is highly recommended after primary or secondline treatment. In particular, several meta-analyzes stress the great prognostic value of EOT PET for predicting evolution after autologous stem cell transplantation $(15,16)$. PET/CT is strongly discouraged in the follow-up of patients with DLBCL according to Lugano 2014 recommendations.

In Follicular Lymphoma (FL) The detection of hypermetabolic foci with high uptake intensity in a patient with indolent NHL should raise suspicion of histological transformation to a more aggressive NHL variant and warrants for a diagnostic biopsy. The changes in stage revealed by the staging PET/CT are of special relevance in those patients with suspected localized disease, in whom radiotherapy is the indication for treatment. Regarding extra nodal disease, PET/CT is able to detect a greater number of affected locations compared to Contrast-enhanced CT (CeCT). PET cannot substitute the BMP performed for staging purpose (15); the latter remains the most sensitive tool to detect bone marrow invasion by disease. An iPET is not currently recommended in FL $(10,11)$. PET CT should not be used as a follow-up technique in patients with indolent lymphomas, with the notable exception of cases with a clinical suspicion of histological transformation. EOT-PET is the recommended technique for evaluating the response at the end of treatment.

Mantle Cell Lymphoma (MCL) is FDG avid in most cases and PET-CT is indicated in the initial staging, which must be supplemented with BMP (being essential in those cases with negative PET-CT) and in some cases with digestive endoscopy, more frequently colonoscopy. At the present time, there is insufficient evidence to recommend PET mid-treatment in patients with MCL. PET is suggested at the end of treatment, with a high negative predictive value, and has 
prognostic value. As for other lymphoma subsets, the use of PET-CT is currently not recommended for the follow-up of patients with $\operatorname{MCL}(10,11)$.

In Hodgkin Lymphoma (HL), baseline PET-CT makes it possible to omit the systematic performance of BMP $(10,11)$, and this is included in the normative in Uruguay. Interim-PET, fundamentally after the second cycle, has a great prognostic value. In limited-stage HL, a positive iPET (DS 4,5) justifies the escalation of treatment; a negative iPET (DS 1-3) supports the decision to omit. In advanced $\mathrm{HL}$, the result of the interim PET allows to adapt the treatment-a negative iPET justifies de-escalation if treatment was started with BEACOPP escalated, and a positive iPET warrants escalation of treatment. In case of CT-detected residual mass, with no proven no FDG-avidity, no more treatment is required. In patients with a positive PET mass / lesion, biopsy can be recommended before considering other treatment options such as radiotherapy. Achieving a negative PET should be the goal of salvage therapy. The complete metabolic response before autologous bone marrow transplantation is associated with a better clinical outcome. PET-CT for follow-up is currently not recommended for the follow-up of the HL in CR after first-line treatment.

\section{PET/CT IMAGING FOR HODGKIN LYMPHOMA IN ARGENTINA}

The management of HL has become a success of the modern oncology after the introduction of the combined modality treatment of radiotherapy with chemotherapy. In 1987, Gianni Bonnadonna and his group established the superiority of ABVD over MOPP, which was the standard treatment for HL before ABVD. Later, the German group introduced a more intensive but also more toxic treatment, the BEACOPP escalated regimen, and a heated debate started on the management of advanced stage HL: ABVD vs. BEACOPP.

For decades, this debate has been the main object of scientific discussion among several cooperative working groups. Since 2005, the introduction of FDGPET/CT for treatment response assessment in HL opened new frontiers or research. PET was originally proposed for end-of-therapy treatment response evaluation and one year later for the early response assessment after few cycles of chemotherapy (iPET) as surrogate test of chemosensitivity. In this clinical setting iPET showed the highest overall accuracy among other prognostic factors to predict ABVD treatment outcome, and a new debate started then: to adapt or not to adapt the treatment to the iPET findings. PET/CT proved to be more sensitive and equally specific to CeCT in HL staging by portraying nodal, and most of all extranodal tumor spread. Based on these new acquisitions, a significant progress was made in HL staging and response assessment, and the installation and use of PET scanners increased all around the world.

In Argentina, the PET story started in 1991. That year, the first PET scanner was installed in the province of Mendoza, with a cyclotron for the production of radiotracers for clinical use. This was the first experience in Latin America, but unfortunately its use was limited by inconstant operativity of the scanner and by its localization in a remote region far from away from urban centers in Argentina. 
Both conditions were a handicap for the patient access to PET scanning and for the distribution of radio-pharmaceutical around the country.

In 2001, the National Atomic Energy Agency (CNEA, Comisión nacional de energía atómica) started the commercial production of FDG at the Ezeiza Atomic Center in the province of Buenos Aires, which made possible the installation of the first PET scanner in the city of Buenos Aires, where the largest number of health care centers in the country have historically been located. In March 2001, the first dedicated stand-alone PET scanner started operating, and fusion images with ceCT scans was performed separately with a dedicated software.

In 2001 we started a difficult journey. The cyclotron had technical limitations, and daily production of the radiopharmaceutical was intermittent, leading to irregularities of FDG supply, which was the only radiotracer produced at that time. This situation caused serious problems in terms of scheduling sessions of PET scanning with an impact on new PET-adapted therapies, which in turn led to conflicts with both patients and referring physicians.

During the first years of its activity the cyclotron production had several interruptions for several reasons, mostly related to malfunction the accelerator engine and also because of a preplanned yearly stop in January for overhauling. Today it is hard to believe that FDG was not available for as long as 30 days and also that the PET scanner frequently suffered from malfunction. Technical problems were solved progressively in both equipment and functioning improved steadily resulting in a more fluid FDG production and a higher reliability of the PET scanner. In 2007, hybrid PET-CT scanners were installed for the first time in Argentina, and they gradually took over the old PET scanners; however, the FDG production was still unable to meet the demand.

In 2009-2010 three private cyclotrons were installed in Buenos Aires that led to a significant increase of FDG supply. At present, more than 25 PET scanners operate in the country. Once the new scanners were installed, the technical skill of the scanner operators, as well as the medical experience of nuclear medicine physicians grew, and this facility expanded in Argentina. The production and supply of FDG became reliable and sustained, and other radiopharmaceuticals were gradually introduced and produced, such as 18F-choline, 18F-DOPA, 68Ga-DOTA-PePeptide, 68Ga-PSMA 18F-NAF, 11C-PIB, and others which are under development at this writing in October 2021. An example of the use of PET/CT before, during, and at the end of treatment of an advanced HL is presented in Figure 1.

It was a long and complex journey. At the beginning, the medical community did not have the minimal information or knowledge on how to use these new diagnostic tools and it took much time to obtain the authorization for reimbursement of the cost of these tracers from the medical insurance companies. During the first years the only PET/CT reimbursed was the EoT-PET while insurances could not cover for the cost of a bPET performed for staging purposes. Later, when both the baseline and the interim PET/CT became more widely used based on the scientific evidence and improved overall HL management, both scans were reimbursed. So, we have been witnessing a progressive growth of the use of this imaging technique in lymphoma and of the tremendous improvement in the daily clinical practice of HL management. The clinical experience and the birth of multidisciplinary teams including radiologists and nuclear medicine physicians associated with the permanent dialogue with hematologists led to a better 


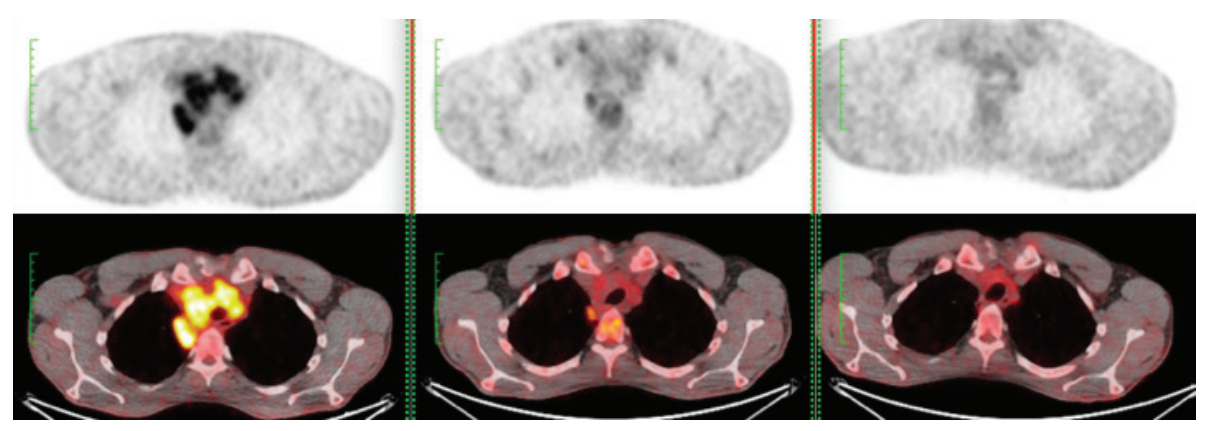

Figure 1. PET/CT. Patient with advanced HL having performed PET/CT at baseline (left), after two cycles of ABVD (center) and after further 4 cycles of esc-BEACOPP (right). The mediastinal lesion, even if decreased in both dimension and uptake show a residual uptake higher than the liver (partial metabolic response, Deauville score 4). Consequently, the patient was treated with esc-BEACOPP with no residual lesion at the end of treatment (complete metabolic response, Deauville score 1).

understanding of the needs for evaluation or response in patients with HL. This in turn, led to improved personalized medical records oriented to the particular clinical status of each patient. In this context, the interest among treating physicians for the clinical research grew exponentially in, and upon launch of new clinical trials in HL, these were considered by the hematologic community the best treatment approach for newly diagnosed HL patients in Argentina.

At the same time, the Argentine Group for the Treatment of Acute Leukemia (GATLA, Grupo argentino para el tratamiento de la leucemia aguda) was growing. GATLA is a non-governmental medical organization of onco-hematologists dedicated to the clinical research for improving the overall treatment of myeloid and lymphoproliferative disorders in children and adults. This group was created in November 1967 by Dr. A. Suarez, Dr. S. Pavlovsky, M. Eppinger-Helft and F. Sackmann Muriel. Since its creation, the lymphoma sub-commission focused on the optimization of the treatment of patients with $\mathrm{HL}$ in order to optimize efficacy and decrease toxicity. However, at this writing in October 2021, PET-CT is still not accessible to all patients throughout Argentina. GATLA representative currently are struggling to ease the implementation of a more homogeneous health care system for all the inhabitants in Argentina, from Salta in the North to Tierra del Fuego in the South.

\section{Interim PET/CT adapted clinical trials for Hodgkin lymphoma in Argentina}

In 1996, the Hodgkin lymphoma sub-commission of GATLA proposed a new clinical trial, aimed to reduce ABVD treatment intensity in HL: briefly, patients with a favorable prognosis (i.e., stage I-IIA) in complete remission (CR) confirmed by a CeCT after 3 ABVD cycles stopped the therapy; and unfavorable group (stage I-IIA slow responders failing CR after 3 cycles of ABVD and stage III-IV) completed their treatment with 3 more ABVD for a total of 6 cycles followed by involved filed radiotherapy (IFRT). $601 \mathrm{HL}$ patients were enrolled: 316 had stage 
III-IV and 285 were favorable patients, 60 (15\%) of this latter (15\%) were slowresponders. The 5-years event-free survival (EFS) of patients entering CR after 3 cycles of ABVD in the favorable group was significantly higher (89\%) with respect to the slow responders (66\%) and to the advanced-stage group (72\%; $P<.001)$ treated with 6 ABVD courses +/- IFRT (17).

After this first experience demonstrating the prognostic relevance of an early complete remission, and thanks to the scientific evidence of the very good outcome of ABVD-treated patients in HL with a negative interim PET and the possibility to easily access PET/CT scanning in Argentina, in 2005 GATLA launched the first PET adapted trial in HL patients, the LH-05 trial (17). This was a prospective, phase 2, non-randomized study conducted in 17 clinical sites in Argentina enrolling adult patients with newly classic HL. The trial was aimed to assess the effectiveness of an interim PET-adapted flexible strategy in all-stage disease. Fourhundred-ninety patients, 300 with early stage (I-II) and 190 with advanced-stage (III-IV) HL were enrolled and treated, whatever their stage, with three ABVD cycles, followed by an iPET (PET3). Patients with a negative PET3 were considered in CR and received no further treatment. Patients with a positive PET3 in CR or PR received three more ABVD cycles followed by IFRT at the dose of 30 Gy to persistent hypermetabolic lesions, patients who were PET3-positive with stable disease (SD) or progressive disease (PD) received further salvage chemotherapy followed by autologous stem cell transplantation (ASCT). All the patients with a positive PET3 treated either with three more ABVD + RT or ASCT underwent final evaluation with an EoT-PET. After a median follow-up of 10 years, 338 out of 490 enrolled patients (69\%) achieved a negative and $152(31 \%)$ a positive PET3. The 5 -years EFS and OS for all patients was $79.2 \%$ and $94.3 \%$, respectively; the 5 -years OS was $97.3 \%$ and $87.3 \%$ for PET3 negative and PET3 positive patients, respectively. Patients with a negative PET3 had a 5-years EFS of $89 \%$ for localized and $80 \%$ for advanced stage, respectively; patients with a positive PET3 reached a 5 -years EFS of 63\% ( $\mathrm{p}<0.0001)$. A multivariate analysis for EFS including age, stage, IPS, bulky disease, extranodal areas and PET3 showed that PET3 status and age were the only parameters correlated to EFS $(\mathrm{p}=0.001$ and 0.046 respectively).

When comparing the results of LH-05 with our previous clinical trial (LH-96) (17) there was no difference in EFS and OS at 5 years but in LH-05 only 31\% received more than 3 cycles of ABVD and IFRT compared to $61 \%$ and $100 \%$ in LH-96, thus demonstrating that an iPET adapted approach reduces exposure to chemo and radiotherapy without compromising the long-term treatment outcome.

However, the long-term follow-up of LH-05 trial demonstrated that there is still room for improvement, in order to identify PET3 negative patients, who ultimately experience disease relapse and escalating treatment in PET3 positive patients, with the intention of improving outcome. GATLA is now designing a new trial with the aim to improve these two different risk groups. New PETderived parameters such as metabolic tumor volume (MTV), total lesion glycolysis (TLG) and radiomics-derived parameters are currently under study to assess their feasibility in Argentina and the possibility to include them in the response assessment in patients enrolled in a new clinical trial on behalf of GATLA. 


\section{PET/CT IMAGING FOR DIFFUSE LARGE B-CELL LYMPHOMA (DLBCL) MANAGEMENT IN UKRAINE}

DLBCL is the most frequent subtype of NHL worldwide and accounts for approximately 30-58\% of all NHL globally by different data registries (18-20). According to Ukrainian Lymphoma Registry and National Cancer Registry, DLBCL account for $24.7 \%$ of all the lymphomas and for $36 \%$ of NHL $(21,22)$. The estimated incidence of DLBCL in Ukraine is 4.2 per 100,000 , compared to 5.6 and 3.8 per 100.000 in the USA and Europe, respectively $(18,19,21)$ Annually, more than 3,500 new cases of NHL are diagnosed in Ukraine (23). However, differently from Western countries in which the 5-year relative survival rate (RSR) is higher than $60 \%$ being as high as $63.9 \%$ in USA (19), in Ukraine this value is much lower, being only $45 \%$. This value should be considered in the context of: (i) in Ukraine the DLBCL affects all age groups, being particularly frequent in older patients (range 19-85, median 51,5 years) (23); and (ii) the average life expectancy in this country is 72 years, which is approximately 10 years lower compared with that recorded in the Western world (24).

\section{Current PET-CT availability and diagnostic approaches for DLBCL in Ukraine}

FDG-PET/CT has been available in Ukraine since 2011 with three active PET/CT centers, two of them being equipped for radiopharmaceuticals production. The first center is located in the "Feofaniya" clinical hospital in Kyiv, equipped with a cyclotron Siemens Eclipse RD and one scanner Siemens Biograph 64. Another center is based in the Kyiv City Oncology Center, and is equipped with a General Electric PET Trace cyclotron and two scanners: a Philips GEMINI TF and a GE Discovery STE. In 2014, another private oncology hospital in Kyiv, the "Lisod", installed a Philips Gemini TF scanner. FDG is the only radiotracer now available for PET scanning in Ukraine. Since all PET/CT scanners are located in Kyiv the accessibility of PET scanning outside the capital is somewhat difficult.

According to the World Health Organization's Global Atlas of Medical Devices, only $4 \%$ of lower-middle income, and 3\% of upper-middle income countries have at least one PET scanner per million people, compared to $29 \%$ of high-income countries $(25,26)$. Unfortunately, Ukraine is not included in this list and at this writing in October 2021, only one PET scanner per approximately 11 million people is available. Importantly, one out of four PET scans performed is for tumor staging in lymphoma patients (27). Nonetheless, in Ukraine PET/CT for lymphoma staging is not routinely performed due to cost and availability constraints. Despite the Ukrainian national guidelines for lymphoma management considering PET/CT mandatory for staging, the following annotation is added: "Provided sufficient funding". As a matter of fact, at this writing in October, the health care program in Ukraine does not cover $100 \%$ of expenses for baseline PET/CT, and in approximately $90 \%$ of the cases the patients have to pay for themselves. As a consequence, one out of five lymphoma patients refuse PET/CT staging for two main reasons: (i) a long period of waiting, which can last for months; and (ii) because of the high cost (in average 500 US dollars), which, according to the official data 
provided by State Statistic Service, corresponds to the average nominal salary of a full-time employee (approximately 490 US dollars) (28).

Despite the above difficulties, over the past 5 years from 2016 to 2020, the number of PET/CT scans performed for DLBCL baseline staging raised from 209 to 370 per year. In addition, the examination frequency per single patient increased over time. The available data from two PET centres show that the highest number of examinations of patients with DLBCL was registered in 2018: 110 in the "Feofaniya" clinical hospital, and 262 in the Kyiv City Oncology Center. This thanks to the special support program provided by Ukrainian Association for patients with Lymphoproliferative Disorders offered during 2018 that covered the cost of PET/CT scanning.

From 2015 to 2017, every new patient admitted underwent PET/CT scanning at least twice: for bPET and EoT-PET. From 2018 onward, a positive tendency to increase the number of PET/CT performed per patient was recorded. Namely, the average number was 2 scans per patient, mostly for bPET and EoT-PET. Thus, in 2019, the breakdown of PET/CT performed in DLBCL patients at baseline, interim and end-of-treatment was 20\%, 3\%, and $77 \%$, respectively.

\section{PET/CT approaches in DLBCL in Ukrainian national guidelines}

Today, diagnosis and treatment of DLBCL in Ukraine is made according to Ukrainian national guidelines, as well as to the constantly updated ESMO, NCCN and other available international recommendations, with adaptation for Ukrainian realities.

Based on the recent Lugano consensus recommendations for lymphoma staging, PET/CT in Ukraine is recommended as the "gold standard" for DLBCL patients staging and post-treatment assessment, with the following warning: PET/ CT at baseline is "recommended", but not "necessarily required" in the absence of financial and technical support. For an interim assessment, we would rather provide CT scan, but iPET/CT imaging could be offered to the patient after 3-4 cycles of therapy in case of suspected disease progression. However, in some difficult clinical cases, an individualized approach outside guideline recommendations is required. For example, PET/CT is not recommended during follow-up, but it could be provided in the suspect of an impending relapse when a specific site of disease could be visualized only by PET/CT scan. An example of a patient with DLBCL treated with RCHOPx6 performing PET at different timepoints of the treatment — at baseline, after 2, and after 6 RCHOP cycles — is given in Figure 2 .

The implementation of the Ukrainian national guidelines for the diagnosis and treatment of lymphoproliferative disorders was the first important step toward a standardized lymphoma management across the country. Since 2009, a lot of painstaking work has been carried out to develop and improve standards for the management of patient affected by DLBCL. In Ukraine, the first patient with DLBCL underwent PET/CT examination in 2011, and since 2014, PET/CT has been included in Ukrainian national guidelines, considering the technical and financial capabilities of its availability and accessibility in Ukraine.

At the moment, the main task of the hematological society in Ukraine is to improve early diagnosis and a standard approach to DLBCL therapy in centers throughout the country to achieve survival rates comparable to the Western world. Despite the fact that currently the availability of PET/CT is limited for 


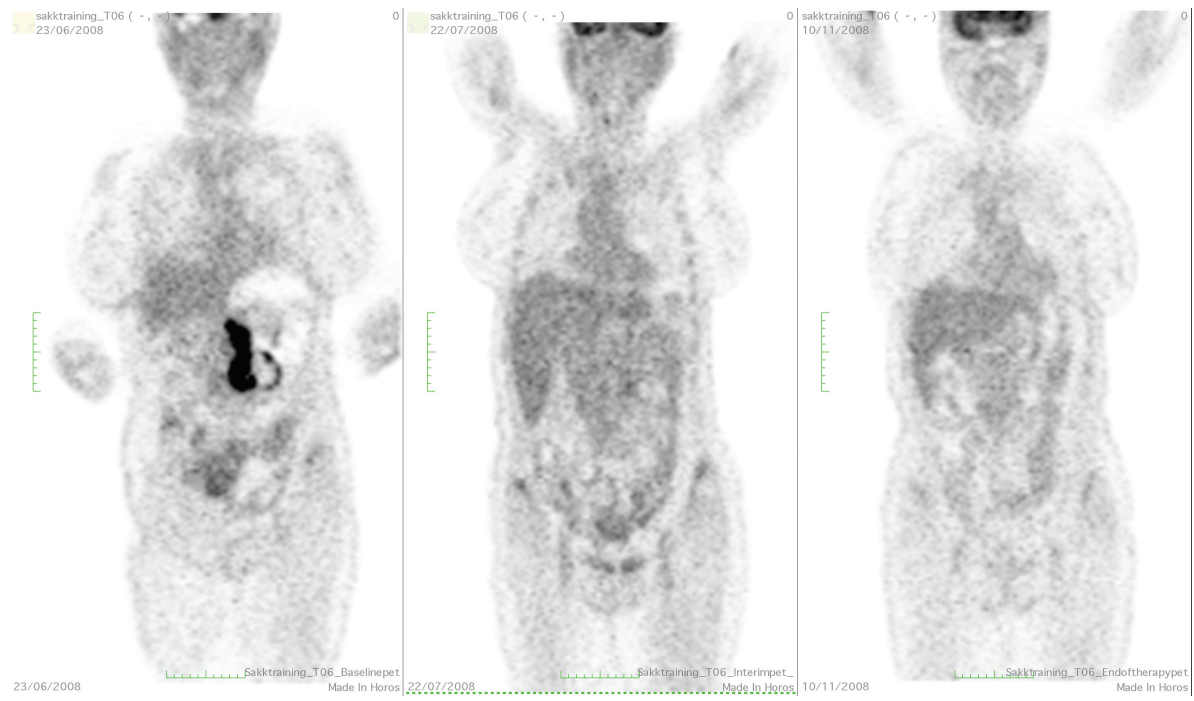

Figure 2. An example of DLBCL lymphoma with PET/CT scan. San performed at baseline (left), after 2 (center), and after 6 R-CHOP cycles, at the end of therapy (right). The uptake in the mesenteric lesions decreased greatly after 2 cycles (while the CT lesion, not shown, had only a small variation) with an uptake comparable to the liver (complete metabolic response with a Deauville score 3 ) and substantially disappear after 6 cycles (complete metabolic response with a Deauville score 1 ).

patients with DLBCL, we are trying to ensure the availability of PET/CT for all patients with DLBCL and other FDG-avid lymphomas with a special focus for baseline, interim and EOT PET/CT. A second task is to implement the close collaboration between hematologists and nuclear medicine specialists for a common PET reporting system, with the aim to answer the clinician questions in terms of disease spread at baseline and response assessment during and after treatment.

However, the main reasons for the slow diffusion of nuclear medicine imaging and, in particular, of PET/CT in Ukraine are two: (i) the insufficient economic stability; and (ii)the bureaucratic over-regulation of the industry in the country. Currently, the state reform of medicine concerns emergency and primary care, and resources have not yet been invested in high-tech development of nuclear medicine diagnostic test at the national level. Today, private business is showing significant interest for investing in PET program development in Ukraine, because of the low offer of PET scanning to the population by the national healthcare system, with significant development perspectives. However, as expected, some limitations to the private investments in nuclear medicine centers have till now limited this opportunity: duration of construction, lack of personnel, difficulties with the production of a radiopharmaceutical and the lack of reimbursement at the national level.

To date, the Ministry of Health in Ukraine is now at work to update the main industry standards of medical care in oncology. PET/CT will be included in the recommendations for diagnosis in accordance with generally accepted international guidelines. This will be considered in the future when reimbursing in oncology will certainly stimulate the investments of the industry. 


\section{PET/CT IMAGING FOR FOLLICULAR LYMPHOMA MANAGEMENT IN SAUDI ARABIA}

Follicular lymphoma (FL) is a heterogeneous low-grade B-cell lymphoma, which constitutes 5\% of all hematological malignancies (29), and 30-35\% of NHL. FL is prevalent in middle to older age groups, and mostly located in nodal sites. However, extranodal sites, such as gastrointestinal, skin, thyroid gland, salivary gland, breast, and testis can also be involved (30-32). The majority of the patients (80-85\%) present with advanced stage (32). The rate of histological transformation is 3\% (33). In the Kingdom of Saudi Arabia, out of the 744 patients with a newly diagnosed non-Hodgkin lymphoma in 2011, 69 (9.3\%) had a FL histology, 42 males and 27 females (34). The cases declined to 25 out of 829 newly diagnosed non-Hodgkin lymphoma (3\%), 14 males and 11 females. in 2015 (35). A study done in the eastern region of the country, reported a $6 \%$ prevalence of FL (35), mostly in the 41-60 age group. In general, the incidence of FL in Saudi Arabia is lower than that reported in the western world of 15-20\% (36-38). According to the 2021 Global Cancer Observatory report, the overall incidence of NHL is 1698 (6.1\%) in Saudi Arabia (39).

\section{Follicular lymphoma diagnosis}

The histological diagnosis of FL is made according to the Saudi Lymphoma Group's Clinical Practice Guidelines for Diagnosis, Management and Follow-up $(34,40)$. Briefly, histological grading is made according to the the presence of centroblast (cB) per high-power field (HPF): grade 1 (0-5 cB per HPF), grade 2 (6-15 $\mathrm{cB}$ per $\mathrm{HPF}$ ), grade $3 \mathrm{~A}$ (>15 cB per $\mathrm{HPF}, \mathrm{cB}$ with intermingled centrocytes), and grade 3B (>15 cB per HPF, pure sheets of blasts) (34). An example of different histology in FL patient is shown in Figure 3. The modern hybrid imaging diagnostic modality with PET/CT in a single-shot scan (41) is the recommended diagnostic tool to detect nodal and extranodal disease $(1,42)$. Moreover, a PET/CT is essential to plan involved-site radiotherapy (ISRT) (43). An example of PET/CT before and after treatment in a FL patient is shown in Figure 4.

\section{Follicular lymphoma treatment}

In general, and according to the Saudi Lymphoma Group Guidelines, FL management is mainly based on Ann Arbor stage, but also on disease burden, age, histological grade, related symptoms, associated co-morbidities and patient preference (44).

A PET-CT is recommended to guide lymph node biopsy. The recommended treatment regimens are single agent rituximab, R-CHOP, R-CVP, rituximab alone, fludarabine and mitoxantrone (RFM), rituximab and bendamustine (R-B) or obinutuzumab plus bendamustine (O-B) (34), as follows:

- Stage I and II: curative radiation therapy, with or without associated immunotherapy with Rituximab or chemotherapy

- Stage I-II, non-bulky disease: observation only

- Stage I-II with bulky disease: immunotherapy with or without radiotherapy 

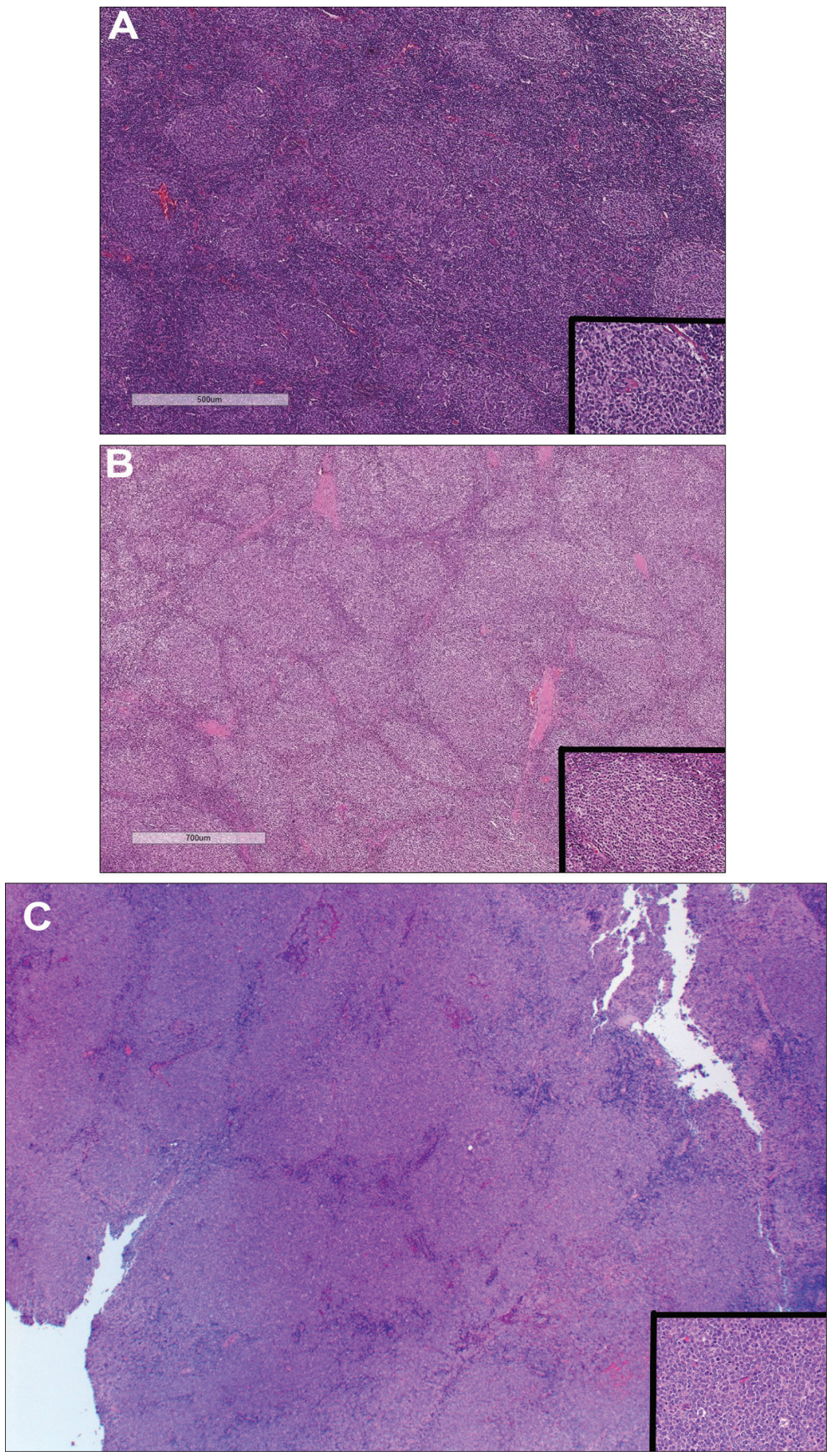

Figure 3. Histology of follicular lymphoma. The WHO Classification utilizes a I-III grading system based on increased numbers of centroblasts counted per high-power field. Grade I-II FL have 0 to 15 centroblasts (follicular mixed). A, Grade III FL has more than 15 centroblasts (follicular large cell). Grade III has been subdivided into grade IIla, in which centrocytes are present, (B) and grade IIIb (C), in which there are sheets of centroblasts (Images courtesy of Saeed Shieban). 

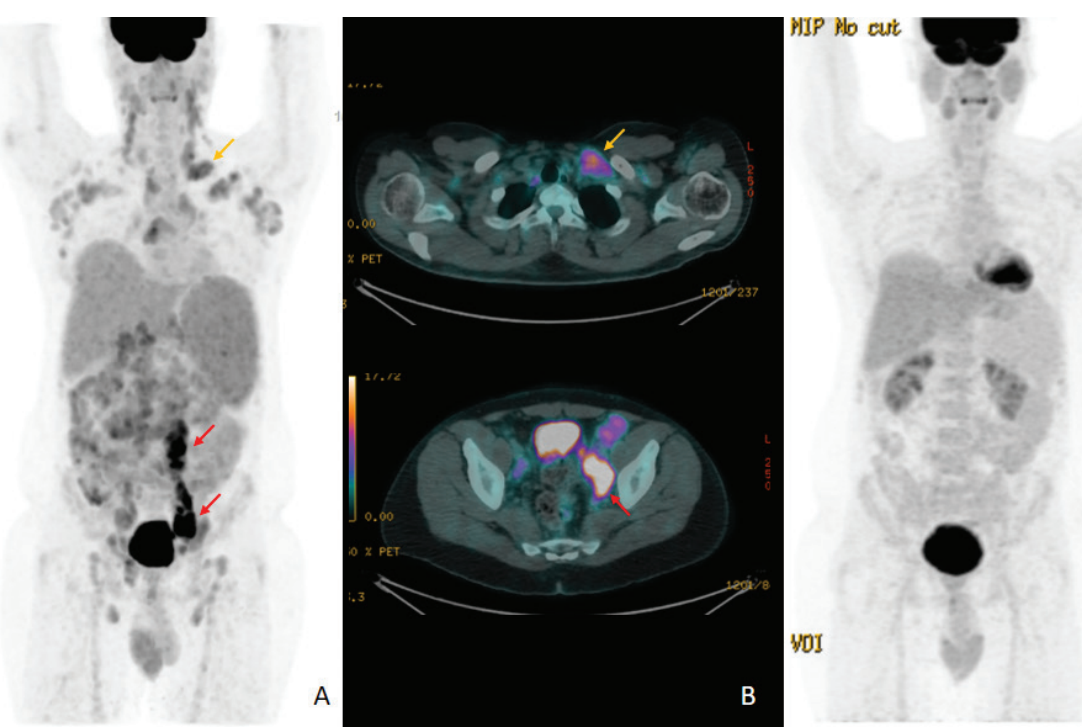

Figure 4. Follicular lymphoma with transformation. A 38-year-old male presented with follicular lymphoma. A, His initial PET/CT showed multiple involved nodes above and below the diaphragm showing moderate FDG uptake. Number of significantly avid nodes seen in the left para-aortic region extending down to the left pelvis, in keeping with transformation. B, Orange arrows show uptake in a left supraclavicular node with an SUV max $_{\text {of }}$ of Red arrows show uptake in left para-aortic and pelvic nodes with SUV $\mathrm{max}_{\max }$ values of up to 11. C, Follow-up PET/CT after treatment showed a complete metabolic response (Images courtesy of Saeed Shieban).

- Grade 3b histology according to World Health Organization (40): combined immunotherapy with rituximab plus cyclophosphamide, vincristine, doxorubicin, and prednisone (R-CHOP)

- Stage III-IV: chemo-immunotherapy with bendamustine and rituximab (RB)

- Allogeneic transplantation: for young patients

- Relapsed or Refractory Disease is based on the indication.

\section{Follicular lymphoma follow-up evaluations}

Post treatment, the patients are addressed to a clinical follow-up every three months for 2 years, then every six months for 3 years, and then annually. The follow-up visits include history, physical examination, complete blood count (CBC), lactate dehydrogenase (LDH), and thyroid-stimulating hormone (TSH). For patients who received radiotherapy to the neck, a CT of neck and a CT of chest, abdomen, and pelvis (CAP) for all the patients along with a mammogram for women once a year after treatment end, and annual influenza immunization (34).

\section{Role of PET/CT imaging in follicular lymphoma}

FL is an FDG-avid disease in more than 95\% of the cases. PET/CT is recommended for baseline staging by international guidelines, as it is able to detect nodal and most of all extranodal disease, including bone marrow, with a superior 
sensitivity (93\%) and specificity compared to a CeCT, thus being able to upstage a significant proportion of the CeCT-staged patients and to ensure a more appropriate treatment for them $(34,45,46)$. PET/CT was also useful to suspect a histological transformation to high-grade lymphoma, based on an elevated value of the maximum Standardized Uptake Value (SUVmax).

FDG-PET/CT is also the best tool to assess treatment response in FL, as a positive end-of-therapy PET is associated with a poor outcome (47). In an ongoing phase 3 trial the HOVON group explored the role of post-induction PET to guide treatment with anti-CD20 in untreated, high-burden FL (48), moving from the assumption that end-of therapy PET/CT has a predictive value on the long-term disease control in FL (49), and it is recommended to assess the response in follicular lymphoma patients (50). Moreover, the total metabolic tumor volume (TMTV) and Tumor lesion glycolysis (TLG) calculated in baseline PET proved also as strong predictive tools on treatment outcome (51). Strategies for PET/CT-guided therapy are currently being assessed by the FOLL-12, FOLL19 and PETReA trials $(48,52)$.

In Saudi Arabia, FL patients are managed and evaluated based on the Saudi Lymphoma guidelines (34). Initial diagnosis is based on the excisional biopsy, and baseline staging is done by PET/CT. Histopathological review is mandatory for all referral cases. End of therapy assessment is done by PET/CT. PET/CT plays a significant role in the staging process of FL, particularly if parameters such as mean tumor volume and TLG are used. In addition, PET/CT is routinely used to guide a biopsy when a transformation is suspected or at the end of therapy for response assessment to the treatment. However, interim adapted PET/CT is not part of daily practice in Saudi Arabia.

\section{Reproducibility of SUV-related indexes for PET/CT reporting in lymphoma}

To assure the highest reliability of the PET/CT reporting by imaging experts, the PET/CT scanner, as any other radiological device, must undergo repeated quality controls, as recommended by national or international guidelines. Moreover, since PET is intrinsically a quantitative imaging method, it could also be calibrated in such a way that the Standardized Uptake Value (SUV), is reproducible within the same PET/CT scanner at different timepoints. SUV is the ratio between the activity measured in a region of interest (ROI) in a tissue or in the tumor and the total activity injected normalized to the patient body weight. Unfortunately, the reproducibility of SUV values across different scanners, that would allow to compare the SUV values in the same patient regardless of the scanner the images were acquired by and to compare the SUV values measured in different patients is low as demonstrated in several multi-centre clinical trials (53-56). The principal limit for using SUV is the error that it associated to its measure. The latter is affected by several factors, some of them related to the patient, others to the procedure for patient preparation and scanning and others to the PET/CT scanner. By adopting a harmonization protocol for PET/CT tumour imaging such as that issued by the European (57) and by the American Societies of Nuclear Medicine (58) and thanks to a program of scanner equalization as the one proposed by the European Association of 
Nuclear Medicine (EANM) $(59,60)$, by the UK PET Clinical Trial Network (CTN) (61), by the American College of Radiology Imaging Network (ACRIN) (62), by the CTN of Society of Nuclear Medicine and Molecular Imaging (SNMMI) $(63,64)$, by the GELTAMO consortium in Spain (65) and by the Italian Foundation of Lymphoma (66) it has been demonstrated that it is possible to achieve PET scan images associated with a SUV variability as low as of $10-20 \%$ (67). Another important factor to consider when comparing images semi-quantitatively is the partial volume effect (PVE). PVE is due to the limited (about $4-5 \mathrm{~mm}$ ) spatial resolution of the PET image system that cause an apparent loss of uptake in a region whose dimension is about 2-3 times the spatial resolution. A $1 \mathrm{~cm}$ diameter lesion with a real uptake with a SUV of 3 would be measured with a SUV of 2. The loss of uptake, and hence of sensitivity, when decreasing the lesion size is described by the recovery curves shown in Figure 5. Modern reconstruction algorithms mitigate this effect modelling the noise and the signal (68) giving the possibility to the user to tune some parameters of the algorithm in order to obtain an image that could be reported with the best accuracy by the nuclear medicine physician. But this tuning will affect the reproducibility of SUV for small lesions across different scanner. Consequently, to compare images across different scanners, the tuning shall produce recovery curve that are within an acceptable range, as the one depicted in dashed line in Figure 5. A simple and complimentary recommendations for PET/CT sites would be to always reconstruct the images in two ways: the one used in medical practice (that is often the one with the "best" reconstruction algorithm available) and a harmonized one, to match the recovery curve limit as shown in Figure 5. These latter could be hence used to compare a subsequent PET/CT done with another scanner with a different technology, at the condition that it is also harmonized.

Should the above recommendations be exported to the daily clinical practice, a full comparability of SUV values measured from different scanner could be reached also outside clinical trials. This in turn could allow to scan the patients in any hospital the patients were admitted to for their healthcare. Indeed, common standards help promote the use of PET/CT imaging and increase the value of publications and their contribution to evidence-based medicine and potentially enable the role of semi-quantitative and quantitative image interpretation since the numeric values would be consistent between platforms and institutes that acquire the data. Moreover, it would be possible to exploit the use of quantitative PET metrics as described in another chapter of this book (https://doi.org/10.36255/ exon-publications.lymphoma.2021.radiomics). To understand to what extent quantitative measurements are carried out in nuclear medicine practice we carried out a survey among 22 nuclear medicine centres in Argentina, Bosnia/Herzegovina, Brazil, Croatia, Egypt, Greece, Honk Kong, Indonesia, Israel, Mauritania, Mauritius, Morocco, Nigeria, Saudi Arabia, Slovenia, Venezuela, and Vietnam. Thirteen of them were equipped with PET/CT hybrid scanners: all of them use semi-quantitative parameters such as SUV to compare the scans performed in the same patient at different timepoints, 10 use SUV variation ( $\triangle$ SUV), and 5 declare to report also MTV in specific cases. This is a clear demonstration of the need for performing inter-scanner equalization procedures and demonstrate the reproducibility of semi-quantitative metrics such as $\mathrm{SUV}_{\max }$ in clinical trials and, possibly, in the daily practice. 




Figure 5. Real-life example of a recovery curve (RC) before after tuning. Blue line, before pre-optimization tuning of the reconstruction algorithm; yellow line, post-optimization tuning of the reconstruction algorithm. The dashed line indicates the acceptable range for the RC.

\section{CONCLUSION}

In this chapter, the authors report the PET/CT implementation in lymphoma across different regions or the world outside the western countries. The differences in the approval of PET/CT use in lymphoma by the national regulatory agencies, as well as the coverage of the cost by welfare, the technical program raised by radiotracer production and delivery to PET/CT sites have been thoroughly reported and included in the complex panorama of PET/CT scanning implementation across countries with different economic income. All the contributors agreed that thanks to PET/CT, the overall lymphoma management definitely improved the standard of care of patients affected by this disease, but several technical and economic factors still represent a hurdle to offer this innovative imaging technique to all lymphoma patients in full compliance with standard international guidelines. As a matter of fact, in some cases, onco-hematologists are still obligated by budget constraints to limit the access to PET scanning for their lymphoma patients to one or two scans per patient or even asking the patients for self-financing. For this reason, it would be desirable that the local scientific community could cooperate with international experts to make the local health stakeholders aware on the medical but also social and economic advantages of PET/CT scanning for lymphoma patients in the context of new therapeutic strategies such as PET/CT-driven treatments or selection of patients requiring expensive treatment.

Acknowledgment: Thanks to the CUDIM team from Montevideo, Uruguay, for their contribution with the reported data: Drs. Juan Pablo Gambini, Monica Rodriguez and Andres Damian. SC and FB thank the participants to the 
questionnaire: Konstantinos Dalianis (Hygeia Hospital, Marousi, Greece), Muaadh Alasbahi (Dr. Sulaiman Al Habib Hospital, Riyadh, Saudi Arabia), Andréia Caroline Fischer da Silveira Fischer (Hospital de Clínicas de Porto Alegre, Porto Alegre, Brasil), Khalida Eddaoui (Ibn Sina Hospital, Rabat, Morocco), Rafael Figueiredo Pohlmann Simões (Fundação Cristiano Varella, Muriaé, Brazil), Jorge Silva (Cintilog Diagnósticos LTDA, Niterói, Brazil), Walter Sabalza (Hospital Universitario de Caracas, CMD La Trinidad, Hosp Militar Dr Carlos Arvelo, Caracas, Venezuela), Maimanah Muhamad (Hospital Canselor Tuanku Muhriz, Kuala Lumpur, Malaysia), Anson Ho-Yin Cheung (Hong Kong Baptist Hospital, Hong Kong, China), Amra Skopljak-Beganović (Clinical Centre of University of Sarajevo, Sarajevo, Bosnia and Herzegovina), Sarsawati Baichoo (Jawaharlall Nehru Hospital, Rose Belle, Mauritius), Sigalit Haruz Waschitz (Shamir MC Assaf Harofeh, Beer Yaakov, Israel), Mohamed Lemine DIENG (National Oncology Center of Nouakchott, Nouakchott, Mauritania), Prasetya Widodo (Indonesian National Nuclear Energy Agency, Jakarta, Indonesia), Quan Nguyen Tien (Bac Ninh general hospital, Bac Ninh, Viet Nam), Samah Kamel Fahmy Elshamndy (Sohag University Hospital, Sohag, Egypt), Ahmed Nageb (Aswan Oncology Center, Aswan, Egypt), Luka Jensterle (University Medical Cetre Ljubljana, Ljubljana, Slovenia), Darko Grosev (University Hospital Centre Zagreb, Zagreb, Croatia), José Ignacio Maldonado (Fuesmen Hospital, Mendoza, Argentina), Oluwajenrola Akintomide (Physics Laboratory, Department of Physics, Ondo, Nigeria)

Conflict of interest: The authors declare no potential conflicts of interest with respect to research, authorship and/or publication of this manuscript.

Copyright and permission statement: The author confirms that the materials included in this chapter do not violate copyright laws. Where relevant, appropriate permissions have been obtained from the original copyright holder(s), and all original sources have been appropriately acknowledged or referenced.

\section{REFERENCES}

1. Cheson BD, Fisher RI, Barrington SF, Cavalli F, Schwartz LH, Zucca E, et al. Recommendations for initial evaluation, staging, and response assessment of Hodgkin and non-Hodgkin lymphoma: the Lugano classification. J Clin Oncol. 2014;32(27):3059-68.https://doi.org/10.1200/JCO.2013.54.8800

2. Centro Uruguayo de Imagenología Molecular. http://www.cudim.org/ [Accessed on 15 Nov 2021]

3. Rodríguez Taroco MG, Cuña EG, Pages C, Schelotto M, González-Sprinberg GA, Castillo LA, et al. Prognostic value of imaging markers from 18FDG-PET/CT in paediatric patients with Hodgkin lymphoma. Nucl Med Commun. 2021;42(3):306-314.https://doi.org/10.1097/MNM.0000000000001337

4. Cerci JJ, Etchebehere EC, Nadel H, Brink A, Bal CS, Rangarajan V, et al. Is True Whole-Body 18FFDG PET/CT Required in Pediatric Lymphoma? An IAEA Multicenter Prospective Study. J Nucl Med. 2019;60(8):1087-1093.https://doi.org/10.2967/jnumed.118.222299

5. Sistema Nacional Integrado de Salud. https://www.gub.uy/ministerio-salud-publica/sistema-nacional -integrado-salud. [Accessed on 15 Nov 2021]

6. https://criteria.iadb.org/webinar-CRITERIA-FNR.pdf. [Accessed on 15 Nov 2021]

7. Fondo Nacional del Ahorro. http://www.fnr.gub.uy/que_es_fnr [Accessed on 15 Nov 2021]

8. Sociedad de Hematología del Uruguay. http://www.shu.com.uy/ [Accessed on 15 Nov 2021] 
9. Fondo Nacional del Ahorro. Medicina Altamente Especializada http://www.fnr.gub.uy/sites/default /files/normativas/tecnicas/n_pet_scan.pdf Set.2017 [Accessed on 15 Nov 2021]

10. Guía De Geltamo PET-TC En Linfomas https://www.geltamo.com/images/PDF/Gula_GELTAMO_ PET_2021.pdf. [Accessed on 15 Nov 2021]

11. Barrington SF, Mikhaeel NG, Kostakoglu L, Meignan M, Hutchings M, Müeller SP, et al. Role of imaging in the staging and response assessment of lymphoma: consensus of the International Conference on Malignant Lymphomas Imaging Working Group. J Clin Oncol. 2014;32(27):3048-58.https://doi. org/10.1200/JCO.2013.53.5229

12. Itti E, Lin C, Dupuis J, Paone G, Capacchione D, Rahmouni A, et al. Prognostic value of interim 18F-FDG PET in patients with diffuse large B-Cell lymphoma: SUV-based assessment at 4 cycles of chemotherapy. J Nucl Med. 2009;50(4):527-33.https://doi.org/10.2967/jnumed.108.057703

13. Hertzberg M, Gandhi MK, Trotman J, Butcher B, Taper J, Johnston A, et al. Early treatment intensification with R-ICE and 90Y-ibritumomab tiuxetan (Zevalin)-BEAM stem cell transplantation in patients with high-risk diffuse large B-cell lymphoma patients and positive interim PET after 4 cycles of R-CHOP-14. Haematologica 2017;102(2):356-363.https://doi.org/10.3324/haematol.2016.154039

14. Sehn LH, Hardy ELG, Gill KK, Al-Tourah AJ, Shustik J, Macpherson NA, et al. Phase 2 Trial of Interim PET Scan-Tailored Therapy in Patients with Advanced Stage Diffuse Large B-Cell Lymphoma (DLBCL) in British Columbia (BC). Blood. 2014; 124 (21): 392.https://doi.org/10.1182/blood. V124.21.392.392

15. Poulou LS, Thanos L, Ziakas PD. Unifying the predictive value of pretransplant FDG PET in patients with lymphoma: a review and meta-analysis of published trials. Eur J Nucl Med Mol Imaging. 2010;37(1):156-62.https://doi.org/10.1007/s00259-009-1258-y

16. Terasawa T, Dahabreh IJ, Nihashi T. Fluorine-18-fluorodeoxyglucose positron emission tomography in response assessment before high-dose chemotherapy for lymphoma: a systematic review and metaanalysis. Oncologist. 2010;15(7):750-9.https://doi.org/10.1634/theoncologist.2010-0054

17. Pavlovsky A, Fernandez I, Kurgansky N, Prates V, Zoppegno L, Negri P,et al. PET-adapted therapy after three cycles of ABVD for all stages of Hodgkin lymphoma: results of the GATLA LH-05 trial. Br J Haematol. 2019;185(5):865-873.https://doi.org/10.1111/bjh.15838

18. Tilly H, Gomes da Silva M, Vitolo U, Jack A, Meignan M, Lopez-Guillermo A, et al. Diffuse large B-cell lymphoma (DLBCL): ESMO Clinical Practice Guidelines for diagnosis, treatment and follow-up. Ann Oncol. 2015;26 Suppl 5:v116-25.https://doi.org/10.1093/annonc/mdv304

19. NIH cancer facts. https://seer.cancer.gov/statfacts/html/dlbcl.html [Accessed on 15 Nov 2021]

20. Horvat M, Zadnik V, Južnič Šetina T, Boltežar L, Pahole Goličnik J, Novaković S, et al. Diffuse large B-cell lymphoma: 10 years' real-world clinical experience with rituximab plus cyclophosphamide, doxorubicin, vincristine and prednisolone. Oncol Lett. 2018;15(3):3602-3609.https://doi. org/10.3892/ol.2018.7774

21. Skrypets T, Stepanishyna Y, Galli GR, Manni M, Hubareva A, Tytorenko I, et al. Subtypes of malignant lymphomas in Ukraine, according to 2016 WHO Classification. Preliminary report of the Ukrainian Lymphoma Registry. Hemat Oncol. 2021; 39:18-22.https://doi.org/10.1002/hon.108_2881

22. National Cancer Registry of Ukraine (NCRU), NATIONAL CANCER INSTITUTE OF UKRAINE CANCER IN UKRAINE, 2018 - 2019, Incidence, mortality, activities of oncological service. http:// www.ncru.inf.ua/publications/BULL_21/index_e.htm [Accessed on 15 Nov 2021]

23. National Cancer Registry of Ukraine (NCRU), NATIONAL CANCER INSTITUTE OF UKRAINE CANCER IN UKRAINE, 2018 - 2019, Incidence, mortality, activities of oncological service, incidence by age and sex. http://www.ncru.inf.ua/publications/BULL_22/PDF/ZAHV.pdf [Accessed on 15 Nov 2021]

24. Life expectancy, Oxford Martin School. https://ourworldindata.org/life-expectancy [Accessed on 15 Nov 2021]

25. World Health Organization. Global atlas of medical devices. Geneva: World Health Organization; 2017.

26. Gallach M, Mikhail Lette M, Abdel-Wahab M, Giammarile F, Pellet O, Paez D. Addressing Global Inequities in Positron Emission Tomography-Computed Tomography (PET-CT) for Cancer Management: A Statistical Model to Guide Strategic Planning. Med Sci Monit. 2020;26:e926544. https://doi.org/10.12659/MSM.926544 
27. Gallamini A, Zwarthoed C, Borra A. Positron Emission Tomography (PET) in Oncology. Cancers (Basel). 2014;6(4):1821-89.https://doi.org/10.3390/cancers6041821

28. Basic indicators of social and economic development of Ukraine. https://ukrstat.org/en/operativ/operativ2021/mp/arh_op_e2021.html

29. Carbone A, Roulland S, Gloghini A, Younes A, von Keudell G, López-Guillermo A, et al. Follicular lymphoma. Nat Rev Dis Primers. 2019;5(1):83.https://doi.org/10.1038/s41572-019-0132-x

30. Sorigue M, Sancho JM. Recent landmark studies in follicular lymphoma. Blood Rev. 2019;35:68-80. https://doi.org/10.1016/j.blre.2019.03.006

31. Takata K, Miyata-Takata T, Sato Y, et al: Pathology of follicular lymphoma. J Clin Exp Hematop 2014; 54:3-9.https://doi.org/10.3960/jslrt.54.3

32. Vitolo U, Ferreri AJ, Montoto S. Follicular lymphomas. Crit Rev Oncol Hematol. 2008;66(3):248-61. https://doi.org/10.1016/j.critrevonc.2008.01.014

33. Montoto S, Davies AJ, Matthews J, Calaminici M, Norton AJ, Amess J, et al. Risk and clinical implications of transformation of follicular lymphoma to diffuse large B-cell lymphoma. J Clin Oncol. 2007;25(17):2426-33.https://doi.org/10.1200/JCO.2006.09.3260

34. Dada R, Al-Mansour M, Alhashmi H, Kandil M, Alhejazi A, Sagheir A, et al. Follicular Lymphoma: Saudi Lymphoma Group's Clinical Practice Guidelines for Diagnosis, Management and Follow-up. Saudi J Med Med Sci. 2019;7(3):214-218.https://doi.org/10.4103/sjmms.sjmms_101_19

35. Iqbal Z, Almusawi B, Alhashim F, Ali Aleassa W, Albueisa ZY, Malik A, et al. Demographic and clinical characteristics of lymphoma patients from Al-Ahsa, Saudi Arabia. J Appl Hematol 2020;11:122-125. https://doi.org/10.4103/joah.joah_30_20

36. Friedberg JW, Taylor MD, Cerhan JR, Flowers CR, Dillon H, Farber CM, et al. Follicular lymphoma in the United States: first report of the national LymphoCare study. J Clin Oncol. 2009;27(8):1202-8. https://doi.org/10.1200/JCO.2008.18.1495

37. Flowers CR, Armitage JO. A decade of progress in lymphoma: advances and continuing challenges. Clin Lymphoma Myeloma Leuk. 2010;10(6):414-23.https://doi.org/10.3816/CLML.2010.n.086

38. Ambinder AJ, Shenoy PJ, Malik N, Maggioncalda A, Nastoupil LJ, Flowers CR. Exploring risk factors for follicular lymphoma. Adv Hematol. 2012;2012:626035.https://doi.org/10.1155/2012/626035

39. WHO: The Global Cancer Observatory. 2021 https://gco.iarc.fr [Accessed on 15 Nov 2021]

40. Swerdlow SH, Campo E, Harris NL, Jaffe ES, Pileri SA, Stein H TJ, et al. WHO Classification of Tumours of Haematopoietic and Lymphoid Tissues. WHO Classification of Tumours, Revised 4th Edition, Volume 2.

41. Torres Espallardo I. PET/CT: underlying physics, instrumentation, and advances. Radiologia. 2017:59(5):431-445.https://doi.org/10.1016/j.rx.2016.10.010

42. Brady JL, Binkley MS, Hajj C, et al. Definitive radiotherapy for localized follicular lymphoma staged by 18F-FDG PET-CT: a collaborative study by ILROG. Blood. 2019;133(3):237-245. Blood. 2019;134(3):331.https://doi.org/10.1182/blood.2019001698

43. Dreyling M, Ghielmini M, Rule S, Salles G, Ladetto M, Tonino SH, et al. Newly diagnosed and relapsed follicular lymphoma: ESMO Clinical Practice Guidelines for diagnosis, treatment and follow-up. Ann Oncol. 2021;32(3):298-308.https://doi.org/10.1016/j.annonc.2020.11.008

44. Ardeshna KM, Smith P, Norton A, Hancock BW, Hoskin PJ, MacLennan KA, et al. Long-term effect of a watch and wait policy versus immediate systemic treatment for asymptomatic advanced-stage non-Hodgkin lymphoma: a randomised controlled trial. Lancet. 2003;362(9383):516-22.https://doi. org/10.1016/S0140-6736(03)14110-4

45. Biggi A, Guerra L, Hofman MS. Current status of FDG-PET/CT in staging of adult lymphoma. Clin Transl Imaging 2015; 3:253-269.https://doi.org/10.1007/s40336-015-0127-x

46. Alnouby A, Ibraheem Nasr IM, Ali I, Rezk M. F-18 FDG PET-CT Versus Contrast Enhanced CT in Detection of Extra Nodal Involvement in Patients with Lymphoma. Indian J Nucl Med. 2018;33(3):183-189.

47. Kouroukis CT, Cheung M, Sussman J, Hodgson D FM, S K: The clinical utility of positron emission tomography in the diagnosis, staging, and clinical management of patients with lymphoma:Program in Evidence-based Care PET Recommendation Report No 12. Toronto, 2015. https://www.cancercareontario.ca/en/content/pet-imaging-lymphoma https://gco.iarc.fr [Accessed on 17 Nov 2021] 
48. Pettitt AR, Barrington S, Kalakonda N, Khan U. NCRI PETREA Trial: A phase 3 evaluation of PET-Guided, Response-Adapted Therapy in patients with previously untreated, advanced-stage, high-tumour-burden Follicular Lymphoma. Hematol Oncol 2019;37:67-68.https://doi.org/10.1002/hon.35_2629

49. Strati P, Ahmed MA, Fowler NH, Nastoupil LJ, Samaniego F, Fayad LE, Hagemeister FB, et al. Pretreatment maximum standardized uptake value predicts outcome after frontline therapy in patients with advanced stage follicular lymphoma. Haematologica. 2020;105(7):1907-1913.https://doi. org/10.3324/haematol.2019.230649

50. Trotman J, Luminari S, Boussetta S, Versari A, Dupuis J, Tychyj C, et al. Prognostic value of PET-CT after first-line therapy in patients with follicular lymphoma: a pooled analysis of central scan review in three multicentre studies. Lancet Haematol. 2014;1(1):e17-27.https://doi.org/10.1016/ S2352-3026(14)70008-0

51. Zaucha JM, Chauvie S, Zaucha R, Biggi A, Gallamini A. The role of PET/CT in the modern treatment of Hodgkin lymphoma. Cancer Treat Rev. 2019;77:44-56.https://doi.org/10.1016/j.ctrv.2019.06.002

52. Guerra L, Bergesio F, Versari A, Franceschetto A, Peano S, Fallanca F, et al. Interim analysis of central review of End-Of-Therapy PET in FOLL12 trial for Follicular Lymphoma. Hemat Oncol. 2019; 37:393https://doi.org/10.1002/hon.62_2631

53. Doot RK, Pierce LA 2nd, Byrd D, Elston B, Allberg KC, Kinahan PE. Biases in Multicenter Longitudinal PET Standardized Uptake Value Measurements. Transl Oncol. 2014; 7(1):48-54https:// doi.org/10.1593/tlo.13850

54. Fahey FH, Kinahan PE, Doot RK, Kocak M, Thurston H, Poussaint TY. Variability in PET quantitation within a multicenter consortium. Med Phys. 2010;37(7):3660-6.https://doi.org/10.1118/1.3455705

55. Velasquez LM, Boellaard R, Kollia G, Hayes W, Hoekstra OS, Lammertsma AA, et al. Repeatability of 18F-FDG PET in a multicenter phase I study of patients with advanced gastrointestinal malignancies. J Nucl Med. 2009;50(10):1646-54.https://doi.org/10.2967/jnumed.109.063347

56. Doot RK, Scheuermann JS, Christian PE, Karp JS, Kinahan PE. Instrumentation factors affecting variance and bias of quantifying tracer uptake with PET/CT. Med Phys. 2010;37(11):6035-46.https://doi. org/10.1118/1.3499298

57. Boellaard R, Delgado-Bolton R, Oyen WJ, Giammarile F, Tatsch K, Eschner W, et al. FDG PET/ CT: EANM procedure guidelines for tumour imaging: version 2.0. Eur J Nucl Med Mol Imaging. 2015;42(2):328-54.https://doi.org/10.1007/s00259-014-2961-x

58. Delbeke D, Coleman RE, Guiberteau MJ, Brown ML, Royal HD, Siegel BA, et al. Procedure guideline for tumor imaging with 18F-FDG PET/CT 1.0. J Nucl Med. 2006;47(5):885-95

59. Boellaard R, Hristova I, Ettinger S, Sera T,Stroobants S, Chiti A, et al. EARL FDG-PET/CT accreditation program: Feasibility, overview and results of first 55 successfully accredited sites. J Nucl Med. 2013; 54 (supplement 2) 2052

60. Zijlstra JM, Boellaard R, Hoekstra OS. Interim positron emission tomography scan in multi-center studies: optimization of visual and quantitative assessments. Leuk Lymphoma. 2009;50(11):1748-9. https://doi.org/10.3109/10428190903308049

61. Barrington SF, MacKewn JE, Schleyer P, Marsden PK, Mikhaeel NG, Qian W, et al. Establishment of a UK-wide network to facilitate the acquisition of quality assured FDG-PET data for clinical trials in lymphoma. Ann Oncol. 2011;22(3):739-745.https://doi.org/10.1093/annonc/mdq428

62. Scheuermann JS, Saffer JR, Karp JS, Levering AM, Siegel BA. Qualification of PET scanners for use in multicenter cancer clinical trials: the American College of Radiology Imaging Network experience. J Nucl Med. 2009;50(7):1187-93.https://doi.org/10.2967/jnumed.108.057455

63. Christian P. Use of a precision fillable clinical simulator phantom for PET/CT scanner validation in multi-center clinical trials: the SNM Clinical Trials Network (CTN) Program. J Nucl Med. 2012;53(suppl 1):437

64. Sunderland JJ, Christian PE. Quantitative PET/CT scanner performance characterization based upon the society of nuclear medicine and molecular imaging clinical trials network oncology clinical simulator phantom. J Nucl Med. 2015;56(1):145-52https://doi.org/10.2967/jnumed.114.148056

65. Bergesio F, De Maggi A, Coronado M, Pardal E, Plaza R, Hernández AC, et al. The 18F phantom clinical trials qualification for 18F-FDG-PET scanning adopted by GELTAMO (Grupo Español de Linfomas/Trasplante Autólogo de Médula Ósea). Rev Esp Med Nucl Imagen Mol (Engl Ed). 2021;40(3):149-154.https://doi.org/10.1016/j.remnie.2020.06.011 
66. Chauvie S, Bergesio F, Fioroni F, Brambilla M, Biggi A, Versari A, et al. The (68)Ge phantom-based FDG-PET site qualification program for clinical trials adopted by FIL (Italian Foundation on Lymphoma). Phys Med. 2016;32(5):651-6.https://doi.org/10.1016/j.ejmp.2016.04.004

67. Kostakoglu L, Chauvie S. PET-Derived Quantitative Metrics for Response and Prognosis in Lymphoma. PET Clin. 2019;14(3):317-329.https://doi.org/10.1016/j.cpet.2019.03.002

68. Chauvie S, Bergesio F, De Ponti E, Morzenti S, De Maggi A, Ragazzoni M, et al. The impact of timeof-flight, resolution recovery, and noise modelling in reconstruction algorithms in non-solid-state detectors PET/CT scanners: - multi-centric comparison of activity recovery in a 68Ge phantom. Phys Med. 2020;(75):85-91https://doi.org/10.1016/j.ejmp.2020.06.002 
